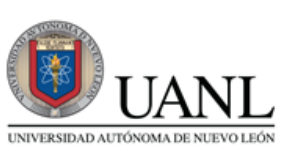

FACPYA
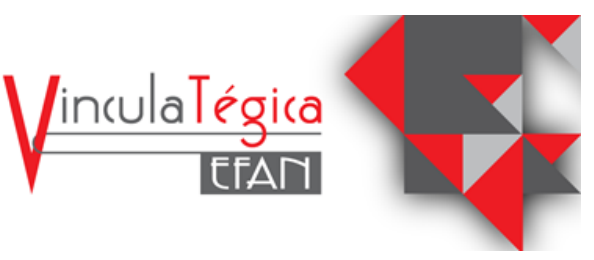

\title{
Estudio comparativo de percepción en seguridad habitacional. El caso de Lomas Residencial y Gaviotas en Poza Rica, Veracruz
}

\author{
Edson Hissait Sampayo García ${ }^{1}$ y Héctor Alejandro Sotelo Acosta ${ }^{2}$ \\ ${ }^{I}$ Estudiante de Posgrado de la Facultad de Arquitectura en la Universidad Autónoma de Nuevo León, \\ edson.sampayog@uanl.edu.mx, Pedro de Alba S/N, Niños Héroes, Ciudad Universitaria, San Nicolás de los \\ Garza, N.L., (818) 4606382 \\ ${ }^{2}$ Profesor e investigador de la Facultad de Arquitectura en la Universidad Autónoma de Nuevo León, \\ martha.ramirezgrz@uanl.edu.mx, Pedro de Alba S/N, Niños Héroes, Ciudad Universitaria, San Nicolás de \\ los Garza, N.L., (818) 4606382
}

Información del artículo revisado por pares

Fecha de aceptación: junio-2021

Fecha de publicación en línea: diciembre-2021

DOI: https://doi.org/10.29105/vtga7.1-118

\section{Resumen}

El presente artículo ofrece un análisis acerca de la percepción de seguridad en los habitantes de dos desarrollos conocidos en la ciudad de Poza Rica, estado de Veracruz. Como en el título se menciona llevan el nombre de Lomas Residencial, y la colonia Gaviotas. Dichos desarrollos se encuentran a no más de 2 kilómetros físicamente de distancia, sin embargo, cuentan con una gran distancia a nivel social y económica entre sus colonos. En esta investigación son tomados en cuenta puntos como arquitectura de los espacios, nivel de ingreso, estudios y sensación de seguridad y la importancia que se le da a esta última necesidad, así como la manera en que se ha resuelto si se ha hecho. También se utilizan métodos cuantitativos (encuesta) casi en la misma proporción de personas en cada desarrollo. Se puede concluir que el nivel de percepción en cuanto a seguridad va muy de la mano con el poder adquisitivo de las familias y el método de compra, sin embargo, la preocupación y necesidad es la misma.

Palabras clave: Clases socioeconómicas, desarrollo habitacional, percepción de inseguridad, Poza Rica, Veracruz.

\begin{abstract}
This article offers an analysis about the perception of security in the inhabitants of two known developments in the city of Poza Rica, state of Veracruz. As mentioned in the title, they bear the name of Lomas Residencial, and the Gaviotas neighborhood. These developments are physically no more than 2 kilometers apart, however, they have a great social and economic distance between their settlers. In this research, points such as architecture of the spaces, income level, studies and sense of security are taken into account and the importance given to this last need, as well as the way in which it has been resolved if it has been done. Quantitative methods (survey) are also used in almost the same proportion of people in each development. It can be concluded that the level of perception regarding security goes very hand in hand with the purchasing power of families and the purchase method, however, the concern and need is the same.
\end{abstract}

Keywords: Socioeconomic classes, housing development, perception of insecurity, Poza Rica, Veracruz.

JEL: J31, D3, D31. 


\section{INTRODUCCIÓN}

Este artículo se realiza en la república mexicana, específicamente el estado de Veracruz, en la ciudad de Poza Rica y se enfoca en presentar un análisis de la percepción de seguridad entre dos desarrollos habitacionales.

El interés en indagar acerca de este fenómeno es hacer un análisis en la forma en que se vive la inseguridad en la vivienda, y su entorno, este mal que afecta a todas las clases sociales y ¿qué relación puede llegar a tener el poder adquisitivo con una mejor percepción de seguridad? También se indaga sobre ¿qué áreas son las más importantes para cualquier persona en su vivienda? Para esto se encuestaron a 106 personas de dos diferentes desarrollos habitacionales en el año 2021 y se hace un análisis de correlación que nos permitirá sustentar la relación que existe entre la situación económica con la sensación de seguridad.

También en este estudio se opta por un análisis cruzado para poder hacer una comparativa como lo indica el título entre los dos grupos de estudio, siendo el desarrollo la variable con la cual se parte la comparativa.

Dado nuestros resultados preliminares hemos podido concluir que se ha optado por mercantilizar un derecho y una cualidad como la seguridad que debería tener toda vivienda como bien menciona el art 25 de la Declaración Universal de Derechos Humanos (1948) que la reconocen como un "Derecho de todas las personas a un nivel adecuado para sí y su familia en alimentación, vestido y vivienda adecuado". La conversión de una necesidad tan básica como lo es la seguridad abre la puerta a la venta de la calidad de vida.

En un contexto histórico la ciudad de Poza Rica, Veracruz es el punto más importante de la zona norte del estado, debido a la gran actividad petrolera y comercial en la región, conociéndose en algún momento como la capital petrolera del país.

En el 2008 con la reforma energética se dio una inyección de capital a la ciudad y área metropolitana debido al proyecto ATG (Activo Integral Aceite Terciario del Golfo) abriendo la puerta a nuevos habitantes de diferentes partes del país y del mundo, dando como consecuencia una gran demanda de vivienda,
Sin embargo a la par de estos 5 años posteriores de crecimiento acelerado, alcanzó a la ciudad los estragos de la "guerra contra el crimen organizado" comenzada en 2006 por el entonces Presidente de la republica Felipe Calderón Hinojosa.

Estas factoras provocaron en la ciudad una nueva forma de ver, proyectar, desarrollar, y construir las viviendas, primeramente, la gran cantidad de inmigración que llegó a la zona trajo como consecuencia una gran demanda de vivienda que la ciudad ni la zona podían proveer. En segundo lugar, la gran inyección de capital y gran fuente de trabajo conllevó a altos niveles de poder adquisitivos, pero de la misma manera a altos índices de desigualdad social, trayendo consigo dos tipos de mercado inmobiliario muy marcado en cuanto a precio de salida se refiere. $\mathrm{Y}$ por último lugar la inseguridad que se vivía en el país, y que también se sufrió en el área creó una sensación y percepción de inseguridad que tendrían que satisfacer los inversionistas y desarrolladores en los nuevos proyectos habitacionales.

En este lapso es cuando se comienza el conjunto habitacional Lomas Residencial, en un entorno cerrado, vigilancia privada, control de acceso, etc. A la par de esto, los fraccionamientos de nivel similar o superior ya existentes adoptaron las mismas medidas de seguridad, a diferencia de las colonias existentes que por el proyecto planteado era imposible controlar su ingreso o delimitar su espacio. Debido a esto la diferencia de plusvalía aumentó y decayó en los conjuntos cerrados y abiertos respectivamente, así como la calidad de vida, la percepción de seguridad y el distanciamiento social entre los diferentes sectores de la población.

\section{MARCO TEÓRICO}

Es sumamente difícil poder medir dicha calidad de vida es posible acercarse sumando varios puntos como estilo de vida, vivienda, satisfacción en la escuela, negocios y en el empleo (Alvarado et al., 2020 y Velarde y Avila, 2002). En el siguiente trabajo ahondamos en el punto de la vivienda y la bondad que puede llegar a tener al generar una sensación de seguridad, o percepción de 
seguridad.

La percepción de inseguridad se refiere a la sensación que puede tener o con la que puede vivir una persona de ser víctima de un delito, sin necesariamente estar en peligro de serlo. O bien como nos dice Vilalta (2009) la perturbación angustiosa del ánimo que se deriva de la diferencia entre el riesgo percibido de ser víctima de un crimen y la victimización de hecho. Otra de las definiciones que se acuñaba a este mal es de Stanko (1995) la preocupación por estar fuera de la casa, probablemente en una zona urbana solo y potencialmente vulnerable a daños personales.

Sin embargo, la problemática ha avanzado a tal magnitud que incluso dentro de la vivienda se puede tener una sensación de inseguridad. La negativa percepción de seguridad en los hogares de nivel medio bajo es fruto de un siempre presente sentimiento a ser y de ser víctima de algún tipo o grupo criminal y si a esto sumamos las deficiencias físicas de la vivienda de nivel medio bajo como lo comenta Galeana (2018). La carencia de servicios básicos ha sido factores determinantes en la construcción del concepto y noción de inseguridad más que de seguridad.

La suma o falta de todos estos factores puede resumirse en la calidad de vida de las personas. Al hablar de esto debemos aclarar que una buena calidad de vida se refiere al bienestar como satisfacción de preferencias y necesidades Nussbaum y Sen (1996), y por último ubicar las necesidades del ser humano, que según Maslow (1943) se dividen en 4 niveles, siendo la seguridad tan importante que solo está por debajo de actividades tan imprescindibles como la respiración, el descanso y la alimentación.

En este artículo no se pretende hablar sobre la probabilidad de robo, exclusión, la distancia, entorno social, la segregación o la desigualdad social, ya que hay varios artículos que hablan sobre estos temas como los de Alvarado, Morales y Ortiz (2020), Alvarado, Valencia e Iturralde (2019), Sánchez (2018) Valverde, Amaral y Ham (1990) y Zapata (1999). Lo que se busca es evidenciar la relación que existe entre el poder adquisitivo y percepción de seguridad, exponer la forma en que se ha comercializado una necesidad básica y que dicha característica pesa por sobre muchos aspectos de la vivienda.

\section{MÉTODO}

Este trabajo es una investigación cuantitativa, aunque lo que se pretende ubicar sea la percepción de los encuestados, la forma de analizar los datos obtenidos es descriptiva y fueron obtenidos mediante una encuesta, la cual se aplicó a 106 personas residentes de los dos desarrollos o conjuntos que son objeto de estudio, todos estos consensos han sido aplicados en el año 2021 con ayuda de la herramienta de Google, enviados mediante la aplicación Whatsapp. e invitaciones por medio de diferentes redes sociales, siendo esta la manera de aplicación para garantizar la seguridad del encuestador y encuestados a causa de la pandemia del Covid que está en curso en el momento de realización de este artículo.

Cabe señalar que la proporción de encuestados no fue en partes iguales (Lomas Residencial con 65 encuestados y Gaviotas con 45) según la localización de su vivienda, como se muestra en la Gráfica1.

Gráfica 1. Encuestados Según su desarrollo

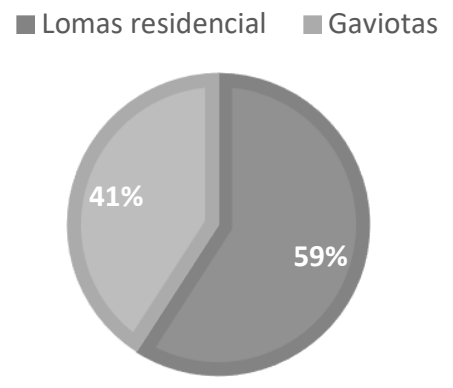

Fuente: Elaboración propia.

Otra parte del instrumento de medición fueron las variables de control. (Tabla 1)

Tabla 1. Variables de control

Variable Definición 
Edad

Género

Escolaridad

Ingreso

Las anteriores variables nos sirven para poder encontrar un patrón entre algunas características de ciertos grupos y poder en algún momento dar una respuesta a los fenómenos que acontecen como lo es la percepción de seguridad.

Se vació la información de las variables de control y se obtuvieron los siguientes resultados mostrados en la Tabla 2. (Es debido mencionar que a la escolaridad se manejaron nomenclaturas del 1 al 4 siendo $1=$ primaria $2=$ secundaria $3=$ preparatoria $4=$ universidad, así también al rango de ingreso siendo del 1 al 5, y son $1=$ menos de $\$ 5,000.00$ pesos $2=$ de $\$ 5,000.00$ a $\$ 10,000.00$ pesos $3=\mathrm{de}$ $\$ 10,000.00$ a $\$ 20,000.00$ pesos $4=\mathrm{de}$
$\$ 20,000.00$ a $\$ 40,000.00$ pesos y $5=$ más de $\$ 40,000.00$ pesos)

Tabla 2. Resultados de variables de control

\begin{tabular}{|c|c|c|c|c|}
\hline Variable & Media & $\begin{array}{l}\text { Desv. } \\
\text { Estanda } \\
r\end{array}$ & $\begin{array}{l}\text { Mínim } \\
\text { o }\end{array}$ & $\begin{array}{c}\text { Máxim } \\
\text { o }\end{array}$ \\
\hline
\end{tabular}

Edad

$\begin{array}{llll}41.98 & 14.454 \quad 15 & 82\end{array}$

Género

$\begin{array}{llll}1.46 & .501 & 1 & 2\end{array}$

Escolarida

d

$\begin{array}{llll}3.61 & .763 & 1 & 4\end{array}$

Ingreso

\begin{tabular}{llll}
3.65 & 1.015 & 1 & 5 \\
\hline Fuente: Elaboración propia
\end{tabular}

Para conocer a fondo y poder resolver las preguntas planteadas en este artículo se redactaron y aplicaron otras variables específicas con el objetivo de establecer las causales en el que una persona se sienta inseguridad en su vivienda y si es esta una necesidad primordial que comparte la población en general o es dependiendo el estrato del cual se procede.

En la siguiente tabla 3 se expone las variables específicas que se resolvieron en la encuesta, al ser aspectos relativos, se procedió a darle un valor numérico a cada punto para un posterior análisis cuantitativo.

Tabla 3. Resultados de variables de control.

\begin{tabular}{cl}
\hline \multicolumn{1}{c}{ Variable } & \multicolumn{1}{c}{ Definición } \\
\hline & $\begin{array}{l}\text { Desarrollo donde se } \\
\text { encuentra la vivienda del } \\
\text { encuestado. Siendo }\end{array}$ \\
Desarrollo & 1= Lomas Residencial \\
& 2= Col. Gaviotas
\end{tabular}




\begin{tabular}{|c|c|}
\hline Seguridad & $\begin{array}{l}\text { Opinión acerca del grado } \\
\text { de seguridad de la } \\
\text { vivienda. } \\
\text { 1= Alto } \\
\text { 2= Medio } \\
3=\text { Malo }\end{array}$ \\
\hline Método & $\begin{array}{l}\text { método de compra de la } \\
\text { vivienda. } \\
1=\text { Compra directa } \\
2=\text { Bancario } \\
3=\text { INFONAVIT } \\
4=\text { FOVISSSTE } 5=\text { Otro }\end{array}$ \\
\hline inversión & $\begin{array}{l}\text { Tipo de inversión } \\
\text { realizada. } 1=\text { Seguridad } \\
2=\text { Acabados } \\
3=\text { Ampliaciones } \\
4=\text { Ninguna de las } \\
\text { anteriores }\end{array}$ \\
\hline Importancia & $\begin{array}{l}\text { Criterios más importantes } \\
\text { en una vivienda. } \\
1=\text { Seguridad } \\
2=\text { Diseño } \\
3=\text { Áreas verdes }\end{array}$ \\
\hline
\end{tabular}

Fuente: Elaboración propia

Al vaciarse los resultados de las anteriores variables obtuvimos los siguientes resultados como se muestra en la tabla 4 .

Tabla 4. Resultados de variables específicas.

\begin{tabular}{lcccc}
\hline Variable & $\begin{array}{c}\text { Medi } \\
\mathrm{a}\end{array}$ & $\begin{array}{c}\text { Desv. } \\
\mathrm{r}\end{array}$ & $\begin{array}{c}\text { Mínim } \\
\mathrm{o}\end{array}$ & $\begin{array}{c}\text { Máxim } \\
\mathrm{o}\end{array}$ \\
\hline Desarrollo & 1.39 & .489 & 1 & 2 \\
Seguridad & 1.92 & .700 & 1 & 3 \\
Método & 2.18 & 1.049 & 1 & 5 \\
inversión & 1.8 & 1.073 & 1 & 4 \\
Importanci & & & & \\
a & 1.18 & .513 & 1 & 3 \\
\hline Fuente: Elaboración propia & & &
\end{tabular}

\section{RESULTADOS}

Anteriormente se explicaron cuáles fueron las variables específicas y de control y la manera en que las abordamos para un posterior análisis. Con base a los resultados obtenidos se muestran algunos patrones muy evidentes al momento de realizar un cruce de variables.

Como se menciona en la introducción de este trabajo de investigación, se pretende desde un inicio establecer y demostrar una relación entre el poder adquisitivo y la percepción de seguridad. Esto lo hicimos comparando dos nichos de diferentes grupos socioeconómicos.

En primer lugar, hay una diferencia muy clara entre los ingresos económicos de ambos desarrollos como se muestra en la siguiente gráfica de barras (Gráfica 2). 
Gráfica 2. Cruce de variables sobre desarrollo e ingreso

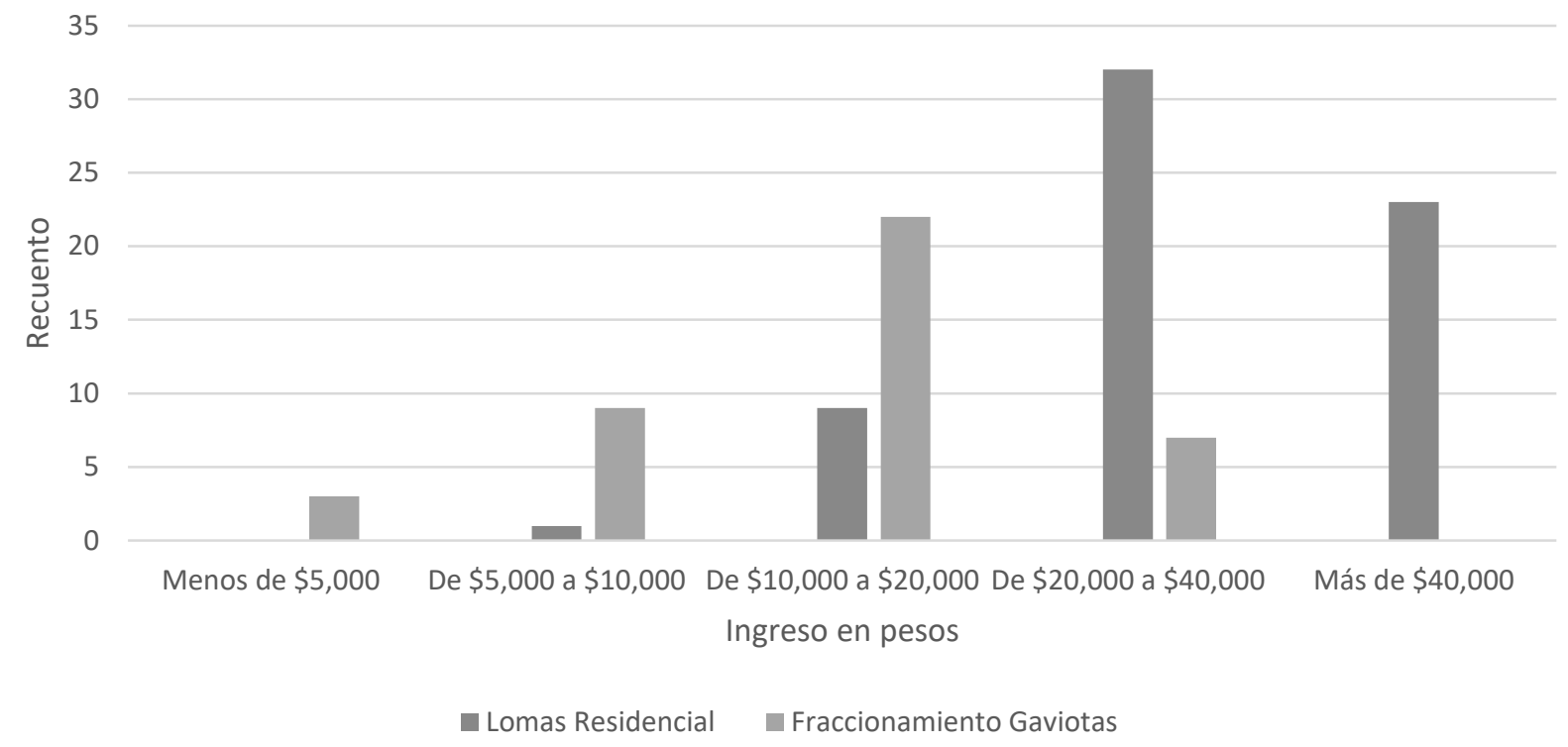

Gráfica 3. Cruce de variables sobre desarrollo e importancia

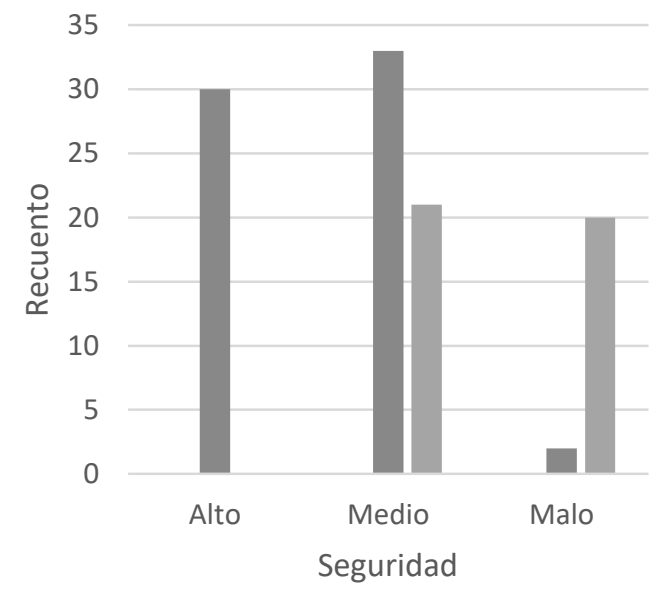

n Lomas Residencial a Fraccionamiento Gaviotas

Fuente: Elaboración propia

Sabiendo la relación que hay entre el desarrollo y el índice de ingreso salarial de sus habitantes procedemos a seguir la comparativa y determinar en qué variables el poder adquisitivo perse tiene impacto en las necesidades y prioridades de los encuestados.

Dentro de las diferencias encontramos una muy importante y cimentación para la realización de este trabajo y es que la percepción de seguridad va de la mano con el desarrollo donde se ubica la vivienda, abriendo

una brecha enorme entre la percepción del habitante de una colonia popular contra el de un habitante de desarrollo privado. Como lo indica la tabla 7. Y por ende podemos deducir que efectivamente el ingreso salarial condiciona la calidad de vida en las personas. Ya que los habitantes de Lomas Residencial mostraron una mejor sensación de seguridad en el desarrollo donde radican.

En los resultados anteriores se ha visto una diferencia muy acentuada entre el ingreso y la percepción de seguridad. Sin embargo, respondiendo a una de las preguntas introductorias para realizar este ejercicio hay tendencias que ignoran cualquier diferencia socioeconómica, como lo son las necesidades básicas del ser humano.

El siguiente análisis es acerca de la importancia que le da a la seguridad el individuo al momento de hacer la compra o de decidirse por establecerse y/o comprar una vivienda. Los resultados fueron muy parejos, realmente las necesidades son las mismas como lo muestra la tabla siguiente (Grafica 4) 
Gráfica 4. Cruce de variables sobre desarrollo y percepción de seguridad.

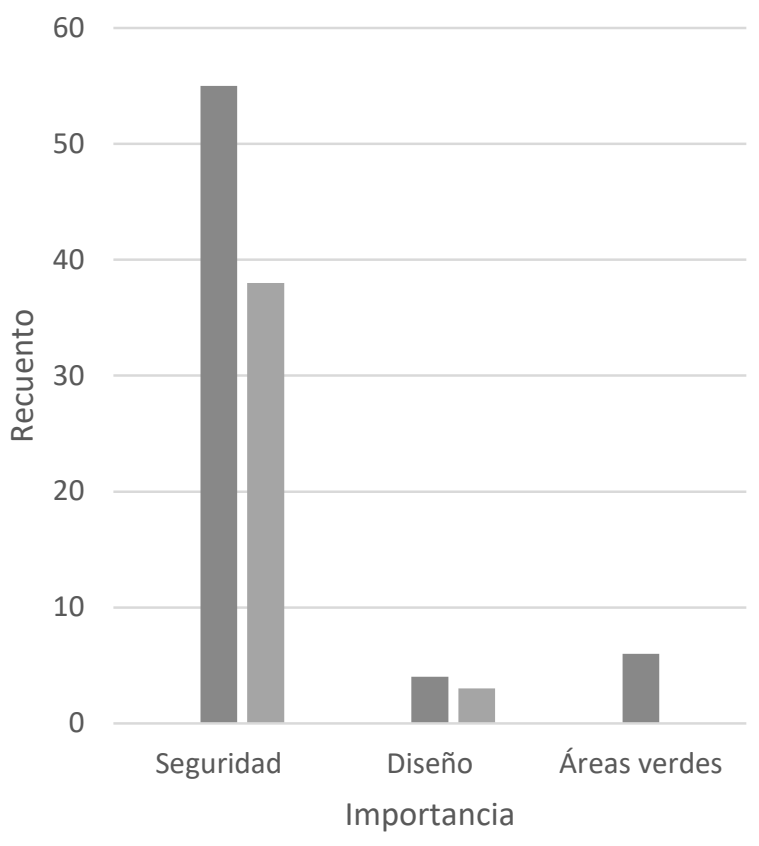

- Lomas Residencial Fraccionamiento Gaviotas

Fuente: Elaboración propia

Otra comparativa que se realizó es en qué invertían los nuevos dueños con la finalidad de mejorar su vivienda, o si lo hacían, y nuevamente la gráfica (Grafica 5) muestra resultados muy similares, a pesar de que el grupo de encuestados que parten de un ingreso salarial más alto tienen la intención de invertir en seguridad, incluso aunque sientan seguro el desarrollo donde habitan. Este fenómeno es algo relevador ya que podemos notar que la seguridad como necesidad es una a la que no se puede llegar a sentirse satisfechos, es una sensación de la que nunca se llega al hartazgo.

Hay una cierta parte de los encuestados que le dieron prioridad al área de acabados, llevando la delantera el desarrollo privado, en el punto de las ampliaciones ambos desarrollos tienen una inclinación parecida y un mayor numero de personas del desarrollo Lomas Residencial señalaron no haber invertido mas allá del desembolso inicial de compra.
Gráfica 5. Cruce de variables sobre desarrollo e importancia.

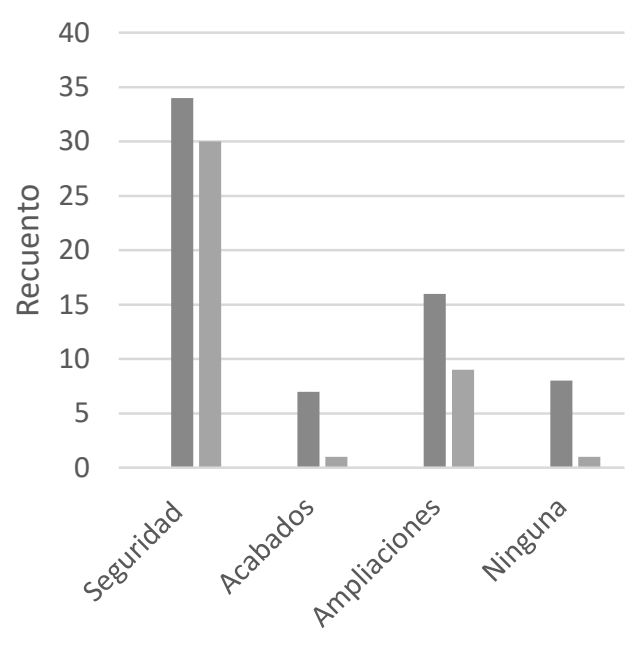

Tipo de inversion

- Lomas Residencial araccionamiento Gaviotas

Fuente: Elaboración propia

El resultado es muy evidente, la seguridad es la cualidad más importante de casi el $90 \%(87.7 \%)$ del total de encuestados. Así que retóricamente hablando ¿por qué los habitantes de Gaviotas habitan un lugar del cual ninguno confirmó como alta su percepción de seguridad habitacional? Pues podemos ver claramente que el factor salarial es necesario hoy en día para establecerse en nichos privados que puedan resolver la problemática que existe en la sociedad.

En cuanto a los resultados de estas dos últimas graficas se puede observar además que es muy mínimo el número de encuestados que consideran otros campos o características de casa habitación como áreas de oportunidad más importantes que la seguridad. Dichas respuestas son de un pequeño grupo de 6 personas que corresponden al $5.7 \%$ de encuestados que le genera más preocupación las áreas verdes, y cabe señalar que solo en el Desarrollo Lomas Residencial hubo esta respuesta, parece que, aunque sea en un número muy bajo, hay un nivel salarial donde se puede permitir que las preocupaciones se vuelvan menos básicas y más del tipo comodidad. 
En cuanto a la importancia del diseño de la vivienda ambos conjuntos tuvieron 4 y 3 encuestados señalándolo como su máxima en cuanto a cualidades habitacionales dando un $3.8 \%$ y $2.8 \%$ respectivamente, siendo una muestra insignificante.

El tipo de compra fungió un factor determinante en el momento de hacer una relación con la seguridad. Los resultados nos permiten inferir que las instituciones públicas, específicamente INFONAVIT tiene requisitos que no van de la mano con la calidad de vida real, son vistas desde el punto de vista conceptual en donde la vivienda es meramente una cubierta y no un refugio.

Entre los diferentes tipos de compra como bancario al casi siempre tratarse de créditos mas altos debido a ingresos mayores pueden optar por elegir entre una mayor variedad de compra.

\section{1 plusvalía}

El hecho de que exista una sensación generalizada en cuanto a la seguridad influye en el valor de la propiedad. El efecto bolo de nieve que cae sobre las casas con ese efecto negativo aumenta año con año, y no permite que los propietarios gocen de un aumento del valor de su propiedad.

En la siguiente imagen (Imagen 1) se puede apreciar la foto de una casa en venta en la colonia Gaviotas.

\section{Imagen 1. Casa ubicada en la Col Gaviotas.}

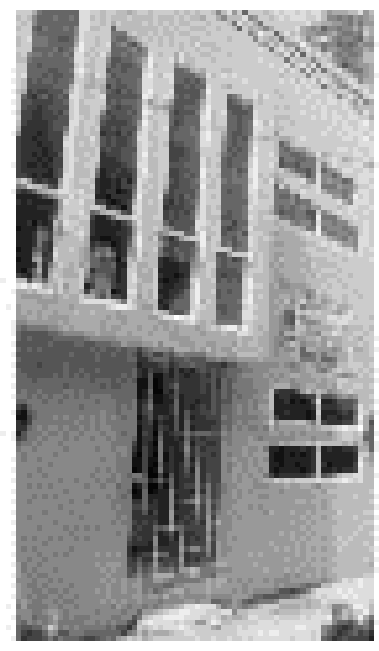

Fuente: casas.trovit.com.mx
La anterior vivienda tiene un precio de $\$ 750,000.00$ pesos contando con un área de construcción de $120 \mathrm{~m}^{2}$ dándonos un aproximado de $\$ 6,000$ pesos el $\mathrm{m}^{2}$.

La siguiente imagen es una casa igualmente de venta ubicada en Lomas Residencial (Imagen 2).

Imagen 2. Casa ubicada en Lomas Residencial

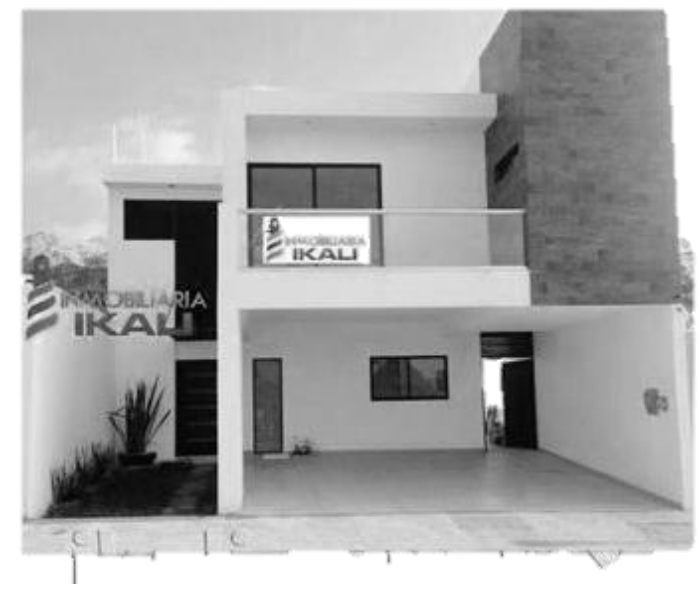

Fuente: propiedades.com

A diferencia de lo que pasa con las casas ubicadas en la colonia Gaviotas, la plusvalía de las viviendas de Lomas Residencial se ha disparado a lo largo de los años, dándole un patrimonio valioso a sus poseedores y un buen negocio para los inversionistas de la ciudad.

La imagen mostrada anteriormente es una casa habitación con un costo de $\$ 3,100,000.00$ y un área de construcción de $250 \mathrm{~m}^{2}$, dándonos un costo por metro de más de $\$ 12,000.00$, ofreciendo valor de casi el doble comparado con su contraparte caso de estudio.

\section{CONCLUSIONES}

De acuerdo con los resultados se puede contestar las preguntas de investigación y procedo a hacerlo.

Hay una relación muy marcada en cuanto al poder adquisitivo y la percepción de seguridad, dicha necesidad se ha convertido en una problemática en la que la iniciativa privada si bien es cierto ha sabido dar solución también se ha dedicado a mercantilizar.

No podemos negar que en los 
desarrollos exclusivos se percibe un ambiente más seguro, sin embargo, hay que entender que esa no es la solución al problema, una de las razones o la más importante es que es un privilegio al que pocas personas pueden acceder, resultando de este un distanciamiento social que es preciso de mención ya que esta tendencia es la que ha venido en aumento.

El cada vez mayor número de Gettos dentro de la mancha urbana, estos grupos de personas que se sienten amenazados por factores como la delincuencia y la inseguridad en general en sus viviendas, ya que no tienen la capacidad económica para comprar su tranquilidad, la cual hoy parece que es un bien privado, recordando con algo de ironía la escena de la película de Iron Man donde el protagonista hace alarde de la llamada privatización de la paz.

Poco a poco la arquitectura habitacional ha llegado a adoptar nuevas tendencias como la arquitectura introspectiva, la cual limita parcial o completamente la vista desde y hacia el interior de la casa, ignorando que por naturaleza somos seres sociables como lo dice la frase del filósofo Aristóteles "El hombre es un ser social por naturaleza". Tampoco podemos adentrarnos en las repercusiones que puede tener este nuevo estilo de vida en nuestra sociedad, pero es seguro que las habrá.

Otro punto importante es que para crear estos desarrollos seguros se opta por apartarse como se menciona en los párrafos anteriores, pero no termina ahí, cada día para sentirse cómodo y protegidos el enclaustre es el camino que hemos tomado, es irónico pensar que para salvaguardarnos hoy en día quien se debe limitar son las posibles víctimas. Es impensable diseñar o construir espacios sin protecciones, lo que termina pareciendo prisiones familiares, y a estas fechas sumando la pandemia que ha impactado no solo el país si no el mundo es casi literal el termino prisión.

Concluyo esta parte haciendo una pequeña crítica y un llamado a todos los sectores que pueden intervenir en cambiar esta situación, el gobierno, las instituciones y la iniciativa privada deberían poder resolver de manera más profunda el problema de la percepción de seguridad. Desde las esferas mas altas que es el gobierno con las políticas publicas el ver que la seguridad sea una necesidad satisfecha en todos los habitantes, un derecho que sea primordial es su agenda política. Una critica a nosotros como arquitectos para retomar los espacios que converjan con el exterior, debemos dejar de pensar que no somos parte del contexto ya que este moldea nuestro estilo de vida.

Así mismo buscar soluciones creativas para poder crear espacios seguros sin que para ello necesitemos aislarnos tras barrotes cual prisioneros, evitar que la tendencia de este siglo sean las casa tipo Bunker.

Sin más que agregar solo quiero recordarnos que todos formamos parte de una sociedad y no podemos seguir permitiendo el distanciamiento a causa de quién puede o no permitirse comprar seguridad o tranquilidad. Este es un derecho universal. 


\section{REFERENCIAS}

Alvarado, E., Morales, D. y J. Ortiz. (2020). Determinantes de la probabilidad de robo a micronegocios en el área metropolitana de Monterrey. Revista de Economía, 37(94), 57-80.

Alvarado, E., Ortiz, J., Martínez, E.S. y O. Iturralde. (2020). El robo a micronegocios y su interrelación con el entorno: un comparativo por género para la zona metropolitana de Monterrey. Revista de El Colegio de San Luis, 10(21), 1-32.

Alvarado, E., Valencia, K. y O. Iturralde. (2019). Impacto del entorno social en el robo a microempresas del área metropolitana de Monterrey. Economía, Sociedad y Territorio, 18(59), 1035-1058.

Selenne. Galeana., (2018). Percepción de seguridad en espacios públicos de conjuntos habitacionales de interés social: Poza Rica y Coatzintla, 2000 al 2016. Revista de urbanismo $n^{\circ} 38$ Universidad de Chile.En: http:// https://auroradechile.uchile.cl/index.php/RU/article/view/47084

Jasso. Lopez., (2013). Percepción de Inseguridad en México. Revista mexicana de opinión publica p. 12-19. En: https://www.sciencedirect.com/science/article/pii/S1870730013723196\#fn0035

Elizabeth A. Stanko, Kathy Hobdell (1993). Assault on men: masculinity anda male victimization, The british journal og criminology en: https://academic.oup.com/bjc/articleabstract/33/3/400/386620

Guevara Daniel, Lobos German, Ortiz S. Elias (2019). Factores determinantes del bienestar financiero y su relación con la calidad de vida en una muestra de profesionales en Guayaquil, Ecuador $\quad$ en: $\quad$ https://scielo.conicyt.cl/scielo.php?script=sci_arttext\&pid=S071807642019000100121

Nussbaum C. Martha, Sen Amartya (1998). La calidad de vida. Fondo de cultura económica en: https://journals.openedition.org/polis/8073

Vilalta, C. (2012). Los determinantes de la percepción de inseguridad frente al delito en México,

Banco Interamericano de Desarrollo en: https://publications.iadb.org/publications/spanish/document/Los-determinantes-de-lapercepción-de-inseguridad-frente-al-delito-en-México.pdf 